\section{Scleroderma-like Pattern in Various Rheumatic Diseases}

\section{To the Editor:}

The recently published paper by van Roon, et al addresses an interesting question about the presence of "scleroderma" pattern in different connective tissue diseases and its association with abnormal pulmonary function tests $^{1}$. The authors have detected it in $88 \%$ of cases with systemic sclerosis (SSc; 35/40), in $17 \%$ of patients with systemic lupus erythematosus (SLE; $5 / 30$ ) and in $13 \%$ of patients with rheumatoid arthritis (RA; $2 / 15$ ). Of note, apart from its association with the presence of Raynaud phenomenon (RP) in connective tissue diseases, "scleroderma-like" capillaroscopic pattern could also be associated with the presence of cutaneous digital vasculitis $\mathrm{s}^{2,3}$.

"Scleroderma/scleroderma-like" is characterized with giant capillaries (diameter $>50 \mu \mathrm{m}$ ), hemorrhages, derangement, avascular areas, and neoangiogenesis. The presence of giant capillaries represents a mandatory criterion in the initial stages of this capillaroscopic pattern that may present as an isolated finding. Scleroderma-type capillaroscopic pattern is observed in the vast majority of patients with SSc $(>90 \%)^{4}$. It appears early in the disease course, facilitates early diagnosis, and is accepted as a diagnostic criterion. Scleroderma-like pattern could be observed in a smaller proportion of patients with other rheumatic diseases (i.e., SLE, RA, and others) ${ }^{1-9}$.

The data from different studies regarding the prevalence of scleroderma-like capillaroscopic changes in different rheumatic diseases as well as the interpretation of the findings vary among different expert groups. In SLE, a number of investigators have reported prevalence of scleroderma-like capillaroscopic changes between $2 \%$ and $9 \%{ }^{4,5,6}$. In 100 patients with SLE, 52 of whom exhibited symptoms of RP, Furtado, et al observed the higher frequency of $15 \%$ of scleroderma-like pattern ${ }^{7}$. An association between scleroderma-like capillaroscopic changes and presence of RP and anti-U1-RNP antibodies has been observed. This stimulated the hypothesis that it could be a manifestation of subclinical overlap between SLE and $\mathrm{SSc}^{7}$, which was accepted among rheumatologists for over a decade. In 2013, it was reported that scleroderma-like pattern could be observed without the presence of overlap with scleroderma and without an association with anti-RNP antibody ${ }^{8}$. It has been detected in $13.3 \%$ of patients with SLE (4/30). The prevalence of RP in the studied patient population was $73 \%(n=22)$ and vasculitis of the peripheral vessels (manifested with periungual erythema and digital erythematous macules) was present in $10 \%(3 / 30)$. All the patients with scleroderma-like capillaroscopic findings exhibited symptoms of secondary RP $(n=4)$ and 2 of them had shown signs of digital vessel vasculitis. Anti-RNP antibody was positive in a single case with secondary RP without vasculitis of peripheral vessels. In all the patients with scleroderma-like capillaroscopic changes, high immunologic activity was found, but signs for overlap with other connective tissue disease were not present ${ }^{3}$. Similarly, van Roon, et al did not find features of overlap with SSc or association with anti-RNP antibody in SLE patients with scleroderma-like pattern ${ }^{1}$.

Diverse data are reported about the presence of capillaroscopic features of microangiopathy in RA. In some of the studies, it has been concluded that a scleroderma-like pattern could not be observed in patients with $\mathrm{RA}^{5,10}$. It has been found that scleroderma-like pattern could be associated with RA and was observed in $14.5 \%$ of cases in a patient population that included 62 patients with RA $(9 / 62$; i.e., 2 patients with peripheral digital vasculitis and 7 patients with secondary RP) ${ }^{8}$. Subsequently, the presence of scleroderma-like pattern in RA has been also reported by other authors. Rajaei, et al and van Roon, et al $^{1}$ have detected such microvascular changes with similar frequency [20.9\% (90/430) and 13\% (2/15), respectively].

Peripheral cutaneous digital vasculitis has been reported as a common feature in SLE and in RA. Thus it should be specifically evaluated, because association with capillaroscopic microangiopathy could be expected. Of note, in SSc, it has been demonstrated that immunosuppressive and vasoactive treatment could improve capillaroscopic findings. It is an intriguing opportunity to predict visceral vascular involvement based on the capillaroscopic features of microangiopathy, but different factors should be taken into account such as disease duration, severity of peripheral vascular syndrome, and the effects of immunosuppressive and vasoactive treatment.

It should be emphasized that scleroderma-like capillaroscopic pattern is not specific for SSc. It could be observed in SLE without being a feature of an overlap syndrome with scleroderma and without association with anti-RNP antibody. Scleroderma-like pattern could be also found in patients with RA without signs of an overlap syndrome. Both in SLE and RA, it is associated not only with RP but also with the presence of digital vasculitis.

SEVDALINA NIKOLOVA LAMBOVA, MD, PhD, Medical University Plovdiv, Faculty of Medicine, Department of Propaedeutics of Internal Diseases, Plovdiv, Bulgaria. Address correspondence to Dr. S.N. Lambova, Medical University - Plovdiv, Department of Propaedeutics of Internal Diseases, 15A Vasil Aprilov Blvd., Plovdiv 4002, Bulgaria. E-mail: sevdalina_n@abv.bg

\section{REFERENCES}

1. Van Roon AM, Huisman CC, van Roon AM, Zhang D, Stel AJ, Smit AJ, et al. Abnormal nailfold capillaroscopy is common in patients with connective tissue disease and associated with abnormal pulmonary function tests. J Rheumatol 2019;46:1109-16.

2. Lambova SN, Müller-Ladner U. Capillaroscopic features of microangiopathy in rheumatoid arthritis patients with peripheral vascular syndrome. Clin Rheumatol 2019;38:2339-41.

3. Lambova SN, Müller-Ladner U. Capillaroscopic pattern in systemic lupus erythematosus and undifferentiated connective tissue disease: What we still have to learn? Rheumatol Int 2013;33:689-95.

4. Maricq HR, LeRoy EC, D'Angelo WA, Medsger TA, Rodnan GP, Sharp GC, et al. Diagnostic potential of in vivo capillary microscopy in scleroderma and related disorders. Arthritis Rheum 1980;23:183-9.

5. Nagy Z, Czirják L. Nailfold digital capillaroscopy in 447 patients with connective tissue disease and Raynaud's disease. J Eur Acad Dermatology Venereol 2004;18:62-8.

6. Kabasakal Y, Elvins DM, Ring EF, McHugh NJ. Quantitative nailfold capillaroscopy findings in a population with connective tissue disease and in normal healthy controls. Ann Rheum Dis 1996;55:507-12.

7. Furtado RNV, Pucinelli MLC, Cristo VV, Andrade LEC, Sato EI. Scleroderma-like nailfold capillaroscopic abnormalities are associated with anti-U1-RNP antibodies and Raynaud's phenomenon in SLE patients. Lupus 2002;11:35-41.

8. Lambova SN, Müller-Ladner U. Capillaroscopic pattern in inflammatory arthritis. Microvasc Res 2012;83:318-22.

9. Rajaei A, Dehghan P, Amiri A. Nailfold capillaroscopy in 430 patients with rheumatoid arthritis. Casp J Intern Med 2017; 8:269-75.

10. Redisch W, Messina EJ, Hughes G, McEwen C. Capillaroscopic observations in rheumatic diseases. Ann Rheum Dis 1970; 29:244-53

First Release April 15 2020; J Rheumatol 2020;47:6; doi: $10.3899 /$ jrheum. 200020 